\title{
Coupling to Devices in Electrically Large Cavities, or Why Classical EMC Evaluation Techniques are Becoming Obsolete
}

\author{
John M. Ladbury \\ NIST 813.02 \\ Boulder, CO, USA \\ ladbury@boulder.nist.gov
}

\author{
Ted H. Lehman \\ Consultant. \\ Belen, NM, USA \\ lehmanth@aol.com
}

\author{
Galen H. Koepke \\ NIST 813.02 \\ Boulder, CO, USA \\ koepke@boulder.nist.gov
}

\begin{abstract}
We show that the coupling between a sensor in an electrically large metallic cavity (artifact) and the outside world is a very complicated problem, with small changes inside the cavity resulting in signal changes of greater than 20 $d B$. However, this problem can be greatly simplified by separating the task into two components: a statistical "reverberation chamber" type component, and a deterministic component (trends as a function of aspect angle, frequency, or other parameter). We give methods for evaluating each of these effects, and show how they can be applied in a specific detailed example.
\end{abstract}

\section{INTRODUCTION}

We exposed an electrically large test artifact (shown in Figure 1 and described below) to an incident plane wave, and measured the coupling to an internal antenna. We evaluated the coupling as a function of frequency, aspect angle, aperture configuration, and internal configuration (boundary conditions). We varied the relative azimuth, roll, and polarization of the artifact and source antenna to give different aspect angles; we covered and uncovered apertures to give different aperture configurations; and we moved a large metal paddle inside the artifact to simulate different boundary conditions. The various paddle positions can simulate effects such as moving circuit cards to different locations in a computer, rolling a metal beverage cart down the aisle of an aircraft, repositioning seats in a

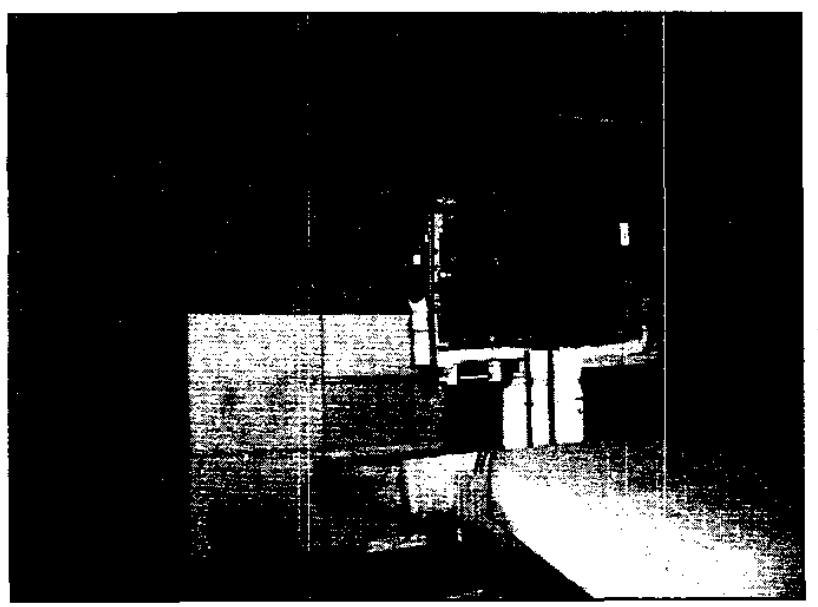

Figure 1. Test artifact and experimental setup.

\section{U.S. Government work not protected by U.S. copyright}

car, or moving a desk in an office. This gave us a flexible generic artifact for simulating several typical operating conditions, although it does not simulate a cavity filled with circuitry or other hardware. This limitation can be addressed in the future if necessary. The results are both very complex (for an individual fixed configuration) and very simple (when viewed statistically).

We begin by describing our test artifact and measurements. We then show examples of the complexity of coupling between an external source and an internal sensor. We conclude that a direct "brute-force" approach is not feasible, and examine the possibility of a statistical approach. We found that the artifact behaved similar to a reverberation chamber (RC), which allowed us to use a number of accepted analytical tools. By averaging over a large number of internal configurations, we were able to evaluate the average pattern characteristics of the artifact, and show that the pattern is quite well behaved. This gives us a combination of statistical and deterministic tools for the analysis of an arbitrary electrically large device.

\section{TEST ARTIFACT}

Our test artifact was an aluminum box, approximately 0.73 $\mathrm{m} \times 0.93 \mathrm{~m} \times 1.03 \mathrm{~m}$ (see Figure 1), designed and fabricated by the Naval Surface Warfare Center, Dahlgren Division (NSWC-DD). Five 1.6-cm-diameter holes were punched at random locations in each of the six sides of the box, for a total of 30 holes. This aperture size was chosen so that all apertures were electrically small at the frequencies we evaluated $(2-4 \mathrm{GHz})$. The holes could be covered with metal tape to give different aperture configurations. The artifact could be rotated about two hubs placed on opposite sides of the box. Inside each hub was an $\mathrm{rf}$ connector. One connector was attached to a dual-ridged horn antenna inside the box, and the other was attached to a shielded $\mathrm{dc}$ motor used for moving an internal paddle.

By placing rotary if joints on each connector, we were able to "roll" the box to different orientations $\rho$ and still maintain signal integrity. By placing the entire setup on a turntable, we could take complete azimuth $\alpha$ cuts at several different "roll" positions. The associated geometry of these measurements is illustrated in Figure 2. 


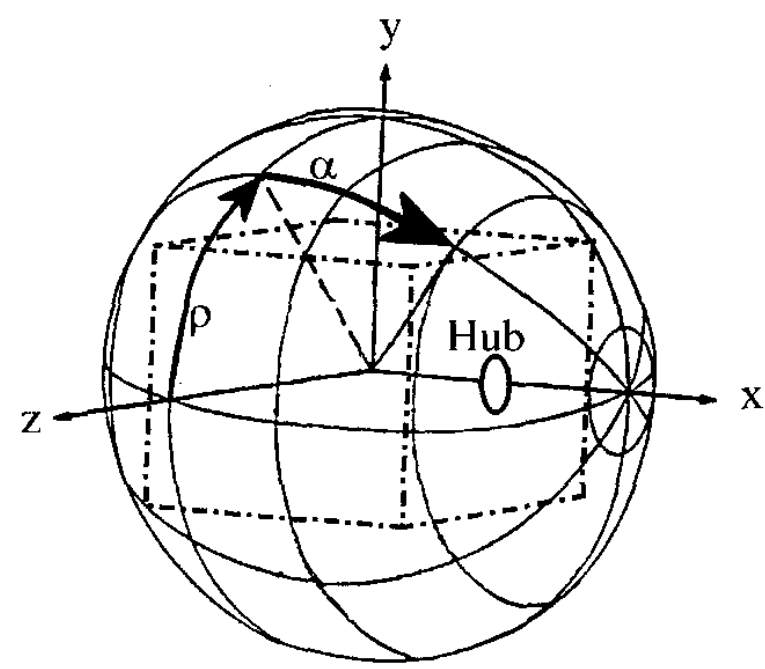

Figure 2. Test artifact coordinate system.

\section{EXPERIMENTAL SETUP}

Our experimental setup consisted of a VNA (vector network analyzer), a low-noise pre-amplifier, a standard gain horn antenna for a signal source, the test artifact described above, and interconnecting cables. For most measurements described here, the VNA was set to scan 801 points over a very narrow frequency span $(100 \mathrm{~Hz})$ in approximately 0.8 seconds (the rotation period of the paddle when turning). Since the frequency span was so narrow, the resulting trace was essentially a plot of received power versus time, or equivalently, received power versus relative paddle position (we could not determine the actual position of the paddle during the measurement). Sweeps were taken and recorded at several different aspect angles (azimuth and roll), and at several frequencies between $2.2 \mathrm{GHz}$ and $4 \mathrm{GHz}$.

We checked the assumption that measurements over a bandwidth of $100 \mathrm{~Hz}$ were similar to $\mathrm{cw}$ measurements over time by taking sweeps at fixed paddle positions. In each case, the readings were essentially constant over the measurement bandwidth. For those measurements in which the paddle was stationary, the coupling was estimated by averaging the 801 samples.

We performed our tests on the NIST spherical scanning range with the center of the box located 8 meters from the aperture of the standard gain horn. Measurements were taken for two polarizations of the horn. Although these polarizations were physically horizontal and vertical, we will refer to them as $\alpha$ polarized and $\rho$ polarized, respectively, to coincide with Figure 2 . In general, our noise floor was approximately $-70 \mathrm{~dB}$.

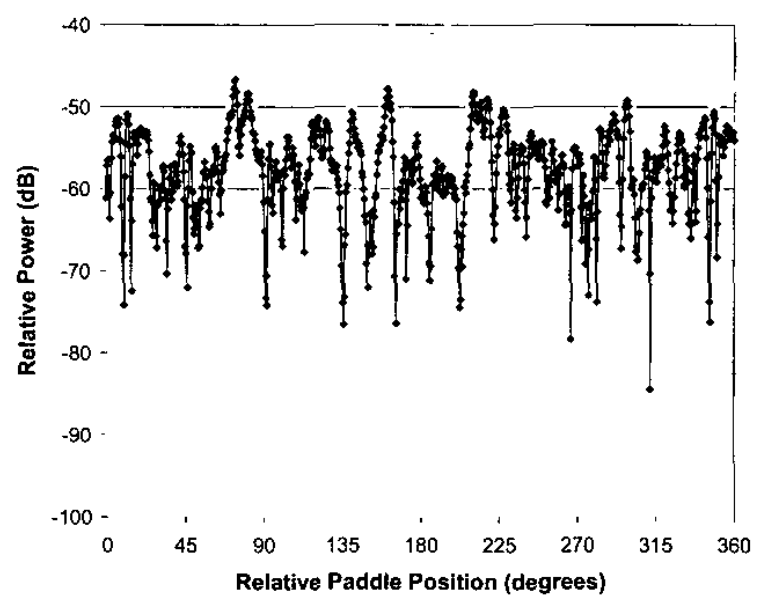

Figure 3. Coupling as a function of paddle position at $3 \mathrm{GHz}$ and a fixed aspect angle. Five holes on each side.

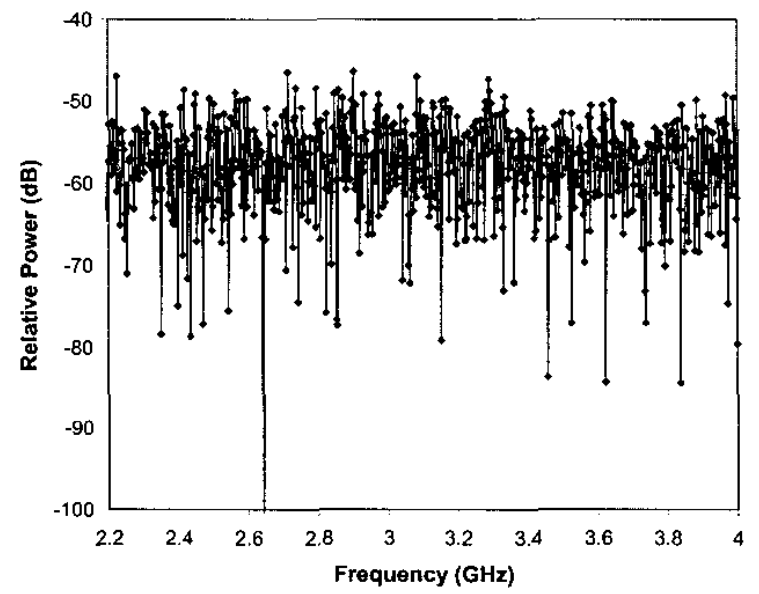

Figure 4. Coupling as a function of frequency at a fixed paddle position and a fixed aspect angle. Five holes on each side.

\section{THE FUNDAMENTAL PROBLEM}

We begin with a few examples showing some of the problems associated with electrically large devices. For all of these measurements, all 30 holes were uncovered. Figure 3 shows a typical plot of coupled power as a function of relative paddle position for a fixed azimuth $\left(0^{\circ}\right)$ and frequency $(3 \mathrm{GHz})$. Figure 4 shows a typical plot of coupled power as a function of frequency for a fixed azimuth $\left(0^{\circ}\right)$ and paddle position. Finally, Figure 5 shows a typical plot of coupled power as a function of azimuth for a fixed frequency $(3 \mathrm{GHz}$ ) and fixed paddle position (unknown but fixed position). In all cases, no corrections have been applied to compensate for cable losses, path losses, or any other systematic trends. These plots simply show the raw measured values of $S_{21}$. This lack of correction was intentional, and will be explained later. 


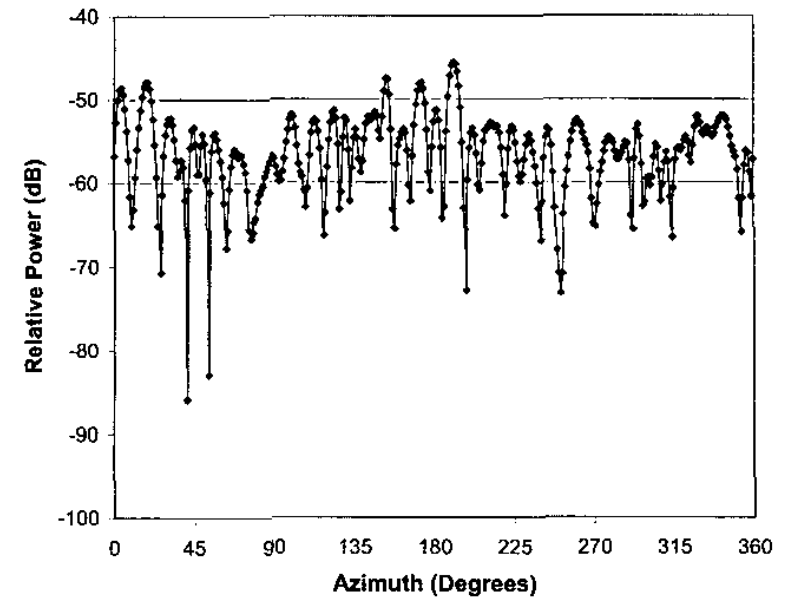

Figure 5. Coupling as a function of azimuth at $3 \mathbf{G H z}$ and a fixed paddle position. Five holes on each side.

In all three sets of data there are large noise-like fluctuations (range $>30 \mathrm{~dB}$, or uncertainties of approximately $+10 /-20 \mathrm{~dB}$ ), and there are no apparent patterns. We can force a pattern by changing the aperture configuration. If we cover all holes on five sides but leave either one or five holes on the remaining side open, we expect this to change the apparent pattern. This is confirmed in Figure 6. Here we can see that the complexity of the data is not reduced when we eliminate all but one hole, and the noise-like characteristics make it difficult to determine where the changed pattern begins or ends. The large fluctuations and the inability to identify trends imply that simple measurement procedures, such as those likely to be included in a standard, will not give reliable or repeatable results.

Surprisingly, the characteristics of Figures 3-5 appear to be similar, with the principal difference being an apparent scale factor on the abscissa. This is similar to the observed behavior of RCs, in which different sampling methods

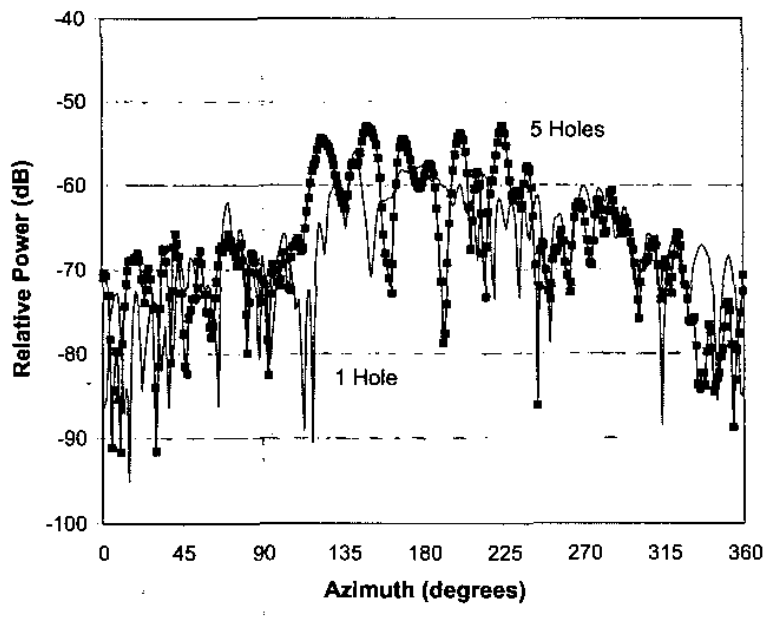

Figure 6. Coupling as a function of azimuth at $3 \mathrm{GHz}$ and a fixed paddle position. Holes on one side only. (sampling as a function of paddle position, source antenna position, sensor position, or, if trends are removed or nonexistent, frequency) can be used interchangeably. It should be no surprise that our artifact behaves like a $\mathrm{RC}$, since it has all of the general characteristics of a RC: an electrically large reflective/conductive cavity. The principal difference is that RC measurements are generally performed with a source inside the cavity, and our measurements were performed with the source outside the cavity.

The similarity between our measurements and RC measurements gives us our first clue as to what problems might be faced when evaluating electrically large objects. Additionally, this similarity implies that some of the tools and procedures used in analyzing RC data can also be applied to this problem.

\section{PROBLEMS AND SOLUTIONS}

Several problems have been encountered in shielded enclosures (SEs), which are essentially the static/deterministic counterparts of RCs (sort of a wicked stepsister). These same problems also apply to our evaluation of an electrically large object. Fortunately, many of the statistical solutions already developed for these problems in RC research can also be applied to problems of coupling with electrically large devices.

\section{Shielded Enclosure Problems}

Some of the greatest challenges that have been faced in SE research are listed below. This is not a complete list, but covers most of the typical problems.

- Individual measurements are essentially worthless. There is little value in a measurement when its uncertainty is greater than $\pm 10 \mathrm{~dB}$.

- Small changes in the setup can make measurements unrepeatable. A repositioned cable or circuit card can completely change the problem.

- We cannot predict "worst-case" configurations. Analytical and numerical models are rarely accurate enough to model all features of a system, resulting in large uncertainties or lengthy tests.

- "True" maxima are very difficult to find.

\section{Sampling and Processing}

In $\mathrm{RCs}$, these problems are generally dealt with using statistical methods. This requires taking multiple measurements (samples) under "similar" but "statistically independent" conditions. "Similar" conditions imply that whatever parameter is changed (boundary conditions, frequency, antenna location), the power coupled to the chamber and loss inside the chamber must remain approximately constant (we don't want to introduce additional variations). "Statistically independent" conditions imply that all changes must be large enough to give a new and independent sample. Changes to boundary conditions, source locations, and sensor locations are most practical because these 
can generally be made without changing the power into or the loss of the chamber. Changes in frequency can also be used, but this is tricky since changes in frequency often result in changes in input power, loss, or both. However, if the functional dependence of input power and loss are known, then this trend can be removed.

Once samples have been taken, they are processed to give some representative value. Typically, either the average or maximum of the samples is computed (but more complicated statistics can also be useful). Averages are much easier to deal with, since sample averages tend to converge to true averages very quickly, even for continuous random processes. Terms such as average, sample average, true average, and expected average can be used interchangeably without much confusion.

Maxima can be much more troublesome due to larger uncertainties and complicated models of continuous random processes. However, some models have been developed for the continuous case [1], and these appear to give satisfactory results.

\section{Solutions Applied to Electrically Large Devices}

From our experience with reverberation chambers, all electrically large low-loss devices (assumed to be cavities) will behave alike; that is, they are statistically equivalent (can be described by the same distributions). Moving the source from inside to outside the device will not improve things; the cavity will behave like a RC if losses and coupling remain constant, or worse if trends or other added variations are present. Pattern effects may cause the average power coupled to the interior of the artifact to vary as the device is rotated (or the source antenna is moved), and trends as a function of frequency become even more complicated because now aperture effects are superimposed on loss effects. In our measurement, we expected both coupling (through holes) and loss (in the artifact walls and the cables) to increase with frequency, approximately canceling each other out. This is why we did not attempt to compensate for frequency trends: the combined effects were small. However, even if frequency and device orientation are held constant, there is no guarantee that the coupled power will remain constant as a function of internal configuration (consider the possibility that something occasionally covers or uncovers an aperture). This can modulate the coupled power and make the problem even worse than a traditional $\mathrm{RC}$ problem. It might seem disturbing to some that the best you can hope for is a reverberation chamber, even if all tests are performed in a perfectly anechoic environment, but there is little that can be done to improve the situation. The problem is in the device, not the environment!

Some experiments have been performed in which the coupling to a wire over a ground plane [2] or to a probe in electronic equipment [3] have been evaluated as a function of aspect angle, and other experiments have evaluated the coupling to an antenna in a cavity as a function of frequency [4]. In all of the experiments, the results showed more variability than would have been expected in a typical $\mathrm{RC}$ measurement, but there was no way to tell whether the increased variability was due to trends (pattern characteristics or frequency-dependent changes in coupling or loss) or to some other unknown effect.

The possible combination of all of these pattern, frequency, and configuration effects makes a general analysis very complicated, and the only hope we have of developing a general measurement technique is to separate the problem into smaller, more manageable pieces. That is, separate out the statistical components from the deterministic trends. The best possible situation would be to find that the statistical components are the same as the statistical components of a $\mathrm{RC}$.

\section{IS THIS A REVERBERATION CHAMBER?}

For our purposes, we will assume that something behaves like a RC if samples taken as a function of paddle position appear to be exponentially distributed. There is no procedure that will definitively prove that something behaves like a RC, or even that a set of samples is exponentially distributed. However, there are a few useful tools and statistics that are used as "quick checks." One of the most popular and powerful of these is the COV, or coefficient of variation, described below. Without the paddle in our artifact, the COV analysis and most of the subsequent analysis would have been difficult or impossible. The only real alternative would have been to move the receiving antenna to different positions, which would have been difficult to implement. This just goes to show: you are really up a creek... without a paddle (sorry).

\section{COEFFICIENT OF VARIATION (COV)}

The coefficient of variation is simply the ratio of the standard deviation to the mean. It is a measure of relative variability (closely related to the variability of a signal on a decibel scale). The power received by an antenna in a reverberation chamber is approximately exponentially distributed [5], and the mean and standard deviation of an exponential distribution are identical. Therefore, the ideal $\mathrm{COV}$ is equal to one. Assuming a sufficient number of samples [6], the sample COV (ratio of the sample standard deviation to the sample mean) should be "close to" the ideal COV of 1.0, with some samples greater than 1.0, and others less. Any results consistently greater than 1.0 indicate additional fluctuations over and above that of a reverberation chamber, and any results consistently less than 1.0 indicates smaller fluctuations than would be expected in a reverberation chamber. In general, we do not expect a sample COV that is consistently less than 1.0 , since this would indicate that the cavity is not operating like a reverberation chamber. To evaluate our artifact, we took 801 samples as a function of paddle position and calculated the sample COV. We repeated this at multiple frequencies, multiple aspect angles, and two different polarizations. Two representative plots give the sample $\mathrm{COV}$ as a func- 
tion of frequency (Figure 7) and aspect angle (Figure 8). Other plots were similar.

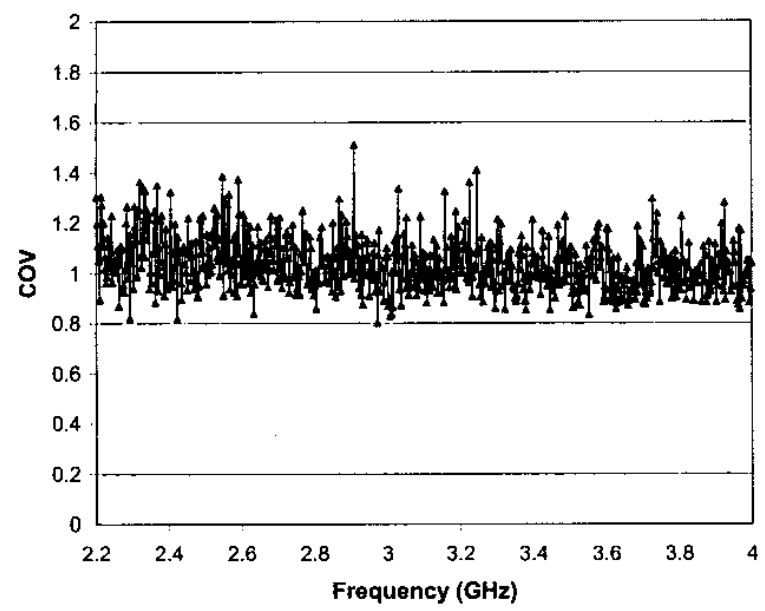

Figure 7. COV as a function of frequency.

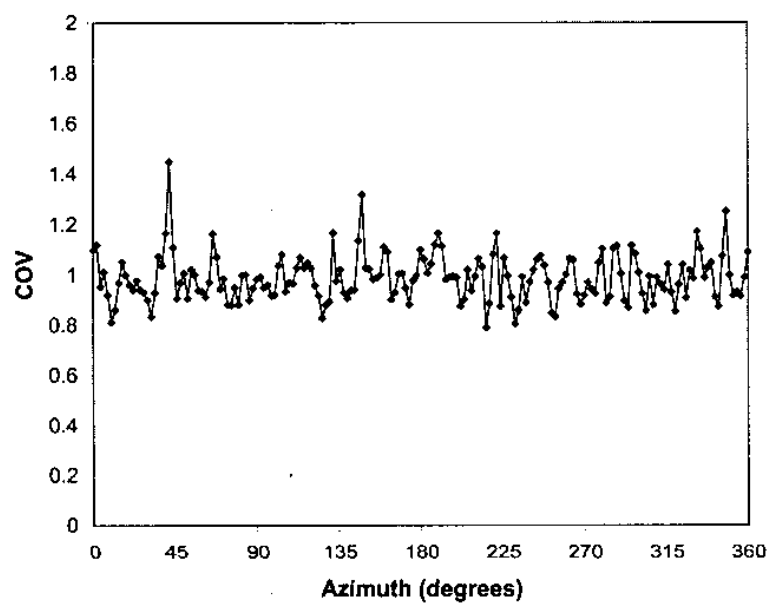

Figure 8. COV as a function of azimuth.

In all cases, the sample COV was close to $1.0( \pm 0.2$ with a few outliers). In Figure 7 there is a slight high bias at low frequencies, indicating that the paddle may be affecting the power coupled to the cavity, but the effect is small. The variability in the $\mathrm{COV}$ is larger than the expected range of \pm 0.07 for 801 independent samples [6]. We think that the additional variability can be traced to one of two sources. First, not all samples were independent, as shown by the correlation of adjacent points in Figure 3. If we assume only 100 independent samples, then the expected variability increases to \pm 0.2 . Second, our test facility was imperfect and was used outside of its recommended distance range, resulting in some environmental effects. This can also increase the variability on the $\mathrm{COV}$ as a function of either frequency or orientation. Based on the COV analysis described above, our artifact appeared to behave like a $\mathrm{RC}$.

\section{DETERMINING TRENDS}

Assuming RC-like behavior, we can assume that the power coupled into the artifact is approximately constant as a function of paddle position for any frequency or aspect angle. We can also assume that the average power received by an antenna inside the artifact will be proportional to the power coupled into the artifact. Therefore, a plot of average received power versus aspect angle should give us an estimate of the true pattern characteristics of the artifact, and a plot of average received power versus frequency should give an estimate of frequency trends. Due to limited space, we will hereafter ignore frequency trends and focus on patterns. Frequency trends can be evaluated in a similar manner.

\section{HOLES ON ONE SIDE}

We covered all holes on five sides of the artifact using copper tape with conductive adhesive. We then evaluated the pattern characteristics when the remaining side (facing the back, $\alpha=180^{\circ}$ ) had five holes open or only one hole open. Hill predicts [7] that the transmission cross section $\sigma_{t}$ (which is proportional to the coupled power) will depend on the polarization and the elevation angle $\theta^{i}$ of the incident field (see Figure 9). For the electric field polarized parallel to the incidence plane (defined by the incident wave vector and the normal to the aperture), we write

$$
\sigma_{t \mathrm{par}} \propto 1+\frac{1}{4} \sin ^{2} \theta^{i}
$$

For perpendicular polarization, we write

$$
\sigma_{t \text { perp }} \propto \cos ^{2} \theta^{i} \text {. }
$$

The proportionality constants are unimportant for our analysis, but are equal in both equations. Therefore, equations (1) and (2) are equal for normal incidence $\left(\theta^{i}=0\right)$.

Equations (1) and (2) assume a single aperture on an infinitely thin, perfectly conducting, infinite plane, but are good approximations to our problem as long as the holes are electrically small, the artifact is electrically large, and the power reradiated by the holes is small relative to the power coupled into the artifact. To check this assumption, we plot the average coupling, as well as predicted curves

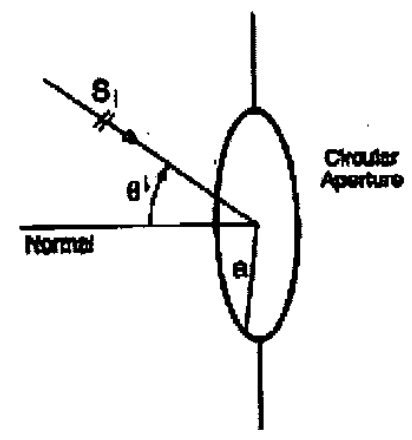

Figure 9. External field incident on a circular aperture. 


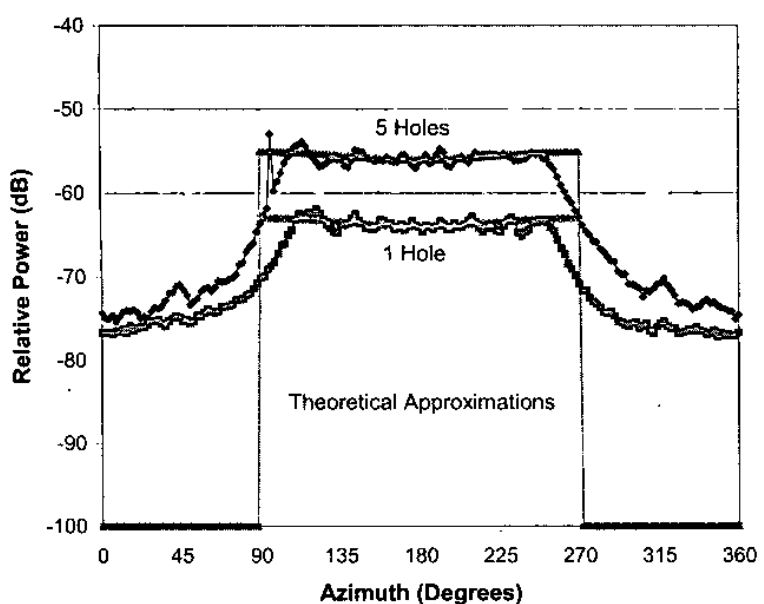

Figure 10. Average coupling through 1 and 5 holes to receiving antenna, measured and predicted, for par. polarization.

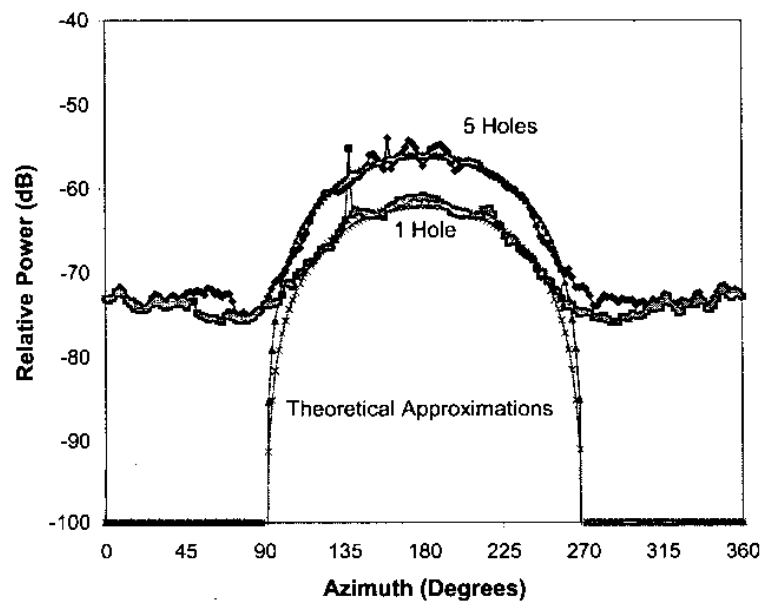

Figure 11. Average coupling through 1 and 5 holes to receiving antenna, measured and predicted, for perp. polarization.

based on equations (1) and (2), as a function of azimuth,

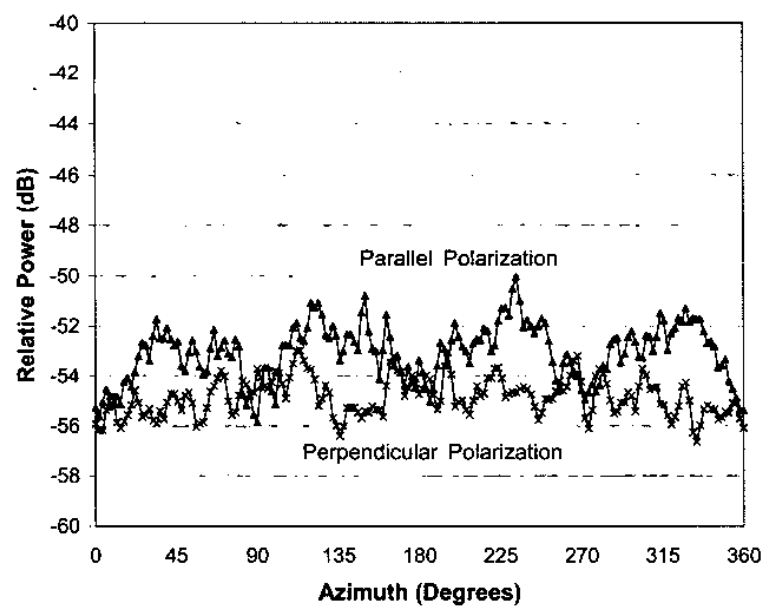

Figure 12. Comparison of coupling for the parallel- and perpendicular polarization cases, all 30 holes open. for both polarizations, and also for one and five holes. The parallel-polarization case, equivalent to the $\alpha$-polarization case for our configuration, is plotted in Figure 10. The perpendicular-polarization case, equivalent to the $\rho$ polarization case for our configuration, is plotted in Figure 11. An offset factor was added to each of the predicted curves to give the best fit between the measured and predicted curves. The agreement between measured data and predictions is quite good, with expected deviations near the noise floor of the VNA. Another difference is that the measured data in Figure 10 appear to begin rolling off sooner than expected and continue rolling off longer than expected. This is most likely due to diffraction effects [8].

Ideally, the only difference between the one-hole curve and the five-hole curve would be a factor of 5, or approximately $7 \mathrm{~dB}$. The actual difference was approximately 8 $\mathrm{dB}$ for the parallel-polarization case, and $6 \mathrm{~dB}$ for the perpendicular-polarization case.

\section{HOLES ON ALL SIDES}

Based on equations (1) and (2), we would expect our artifact to be perfectly isotropic if each side has the same number of identical circular holes (the analysis is tedious, but can be done). Since the perpendicular-polarization case agrees so well with the prediction, we expect that a similar plot for the case when all 30 holes are open should give a nearly constant pattern. However, deviations from the ideal curve in Figure 10 imply that there will also be deviations from the ideal constant curve when all 30 holes are open. For an incident field normal to any surface of the artifact, only holes on that surface will couple an appreciable amount of power. If we increase $\theta^{i}$ by $45^{\circ}$, the coupling through the holes in the original surface remains approximately constant, and coupling through the holes in the "approaching" surface should be comparable. Thus, the power that penetrates the artifact when a corner is illuminated should be greater by a factor of two $(3 \mathrm{~dB})$ than when a single surface is illuminated. However, both the paralleland perpendicular-polarized cases should agree whenever the incident field is normal to one of the surfaces. These characteristics are shown in Figure 12. This figure illustrates one potential problem with simply evaluating immunity characteristics at only four aspect angles: in general, only the four surfaces are illuminated $\left(0^{\circ}, 90^{\circ}, 180^{\circ}\right.$, and $270^{\circ}$ in Figure 12). These happen to be the points of lowest coupling on our artifact.

Unfortunately, the two curves are difficult to differentiate, since the difference between them is on average less than 3 $\mathrm{dB}$, and the statistical uncertainty in the measured data is approximately $\pm 2 \mathrm{~dB}$. We can reduce the uncertainty in the measurement by averaging over frequency. To do this, we average received power in decibels at various frequencies. This approach is better than linear averaging because it allows for different scale factors. Although not theoretically rigorous, this approach gives good qualitative results, 
and with some additional justification may also be quantitatively useful.

As an example, we took data similar to that shown in Figure 12 at ten different frequencies and averaged the results on a decibel scale. This should reduce the measurements uncertainty by a factor of $\sqrt{10}$ or a little more than a factor of 3 . Thus the uncertainty decreases from approximately $\pm 2 \mathrm{~dB}$ to approximately $\pm 0.7 \mathrm{~dB}$. As shown in Figure 14, the results of the averaging illustrate the characteristics of the parallel- and perpendicular-polarization curves much better than the results without averaging shown in Figure 6. The perpendicular-polarization data is approximately flat, and both curves agree at $0^{\circ}, 90^{\circ}, 180^{\circ}$, and $270^{\circ}$, but when the corners are illuminated the parallel polarization shows $2-3 \mathrm{~dB}$ more coupling than the perpendicular polarization.

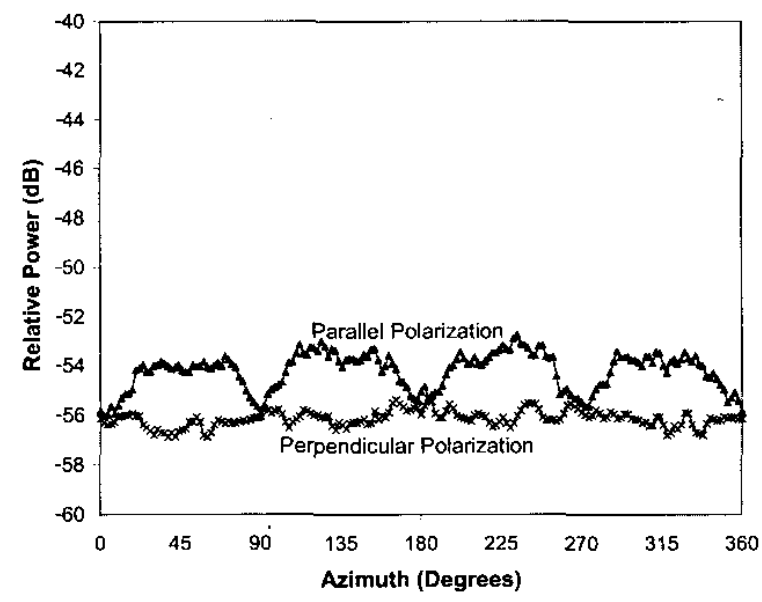

Figure 14. Comparison of coupling for the parallel- and perpendicular polarization cases, all 30 holes open. Averaged over frequency.

\section{DATA IN THREE DIMENSIONS}

Thus far we have focused on simple planar cuts. However, we can easily extend the analysis and measurements to three dimensions. We first approximated the curves shown in Figure 10 and Figure 11 with a simple heuristic function and then predicted what the pattern would look like in three dimensions. We present only the $\alpha$-polarized data here. (We can no longer refer to parallel- or perpendicularpolarizations since we now have apertures on multiple surfaces.) The $\rho$-polarized data showed similar agreement between measurements and predictions. Our prediction is shown in Figure 13. We then evaluated our artifact over 180 azimuth angles (every 2 degrees), 7 roll angles (every 30 degrees), and 10 frequencies $(2.2 \mathrm{GHz}$ to $4 \mathrm{GHz}$, every $0.2 \mathrm{GHz}$ ), and the spherical pattern measured at $3 \mathrm{GHz}$ is shown in Figure 15. All three-dimensional data are plotted assuming that the rotational axis is vertical rather than sideto-side as shown in Figure 2. The data shown here are simi-

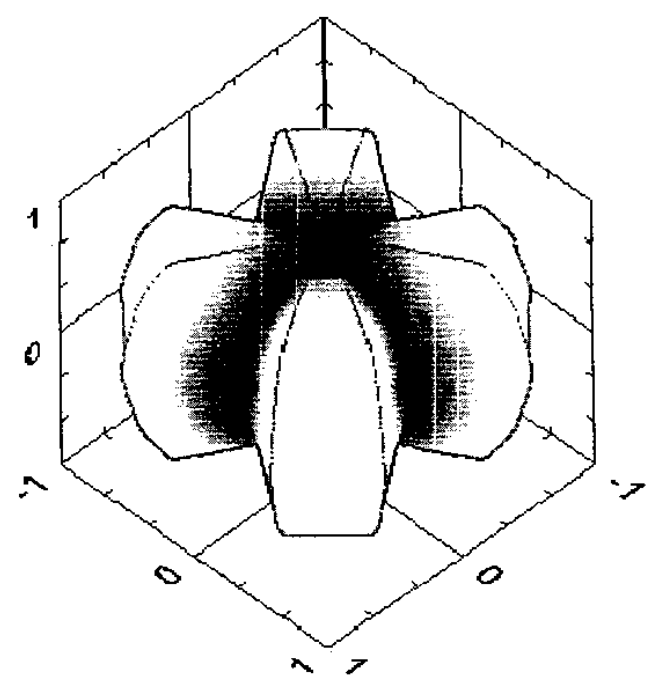

Figure 13. Predicted spherical pattern of our artifact.

lar to that shown in Figure 13, but are much noisier. Even so, the four-lobed structure of the data is still apparent. Finally, we averaged the data over frequency as described above, and the results are shown in Figure 16. The agreement between Figure 13 and Figure 16 is quite good.

\section{INTERPRETATION}

The combination of deterministic and statistical characteristics implies that neither a purely deterministic approach nor a purely statistical approach is appropriate for evaluating an electrically large device. A purely statistical approach, in which a large number of measurements are taken as a function of aspect angle or frequency, combined, and the data analyzed statistically, will tend to overestimate the variability of the system because trends and random fluc-

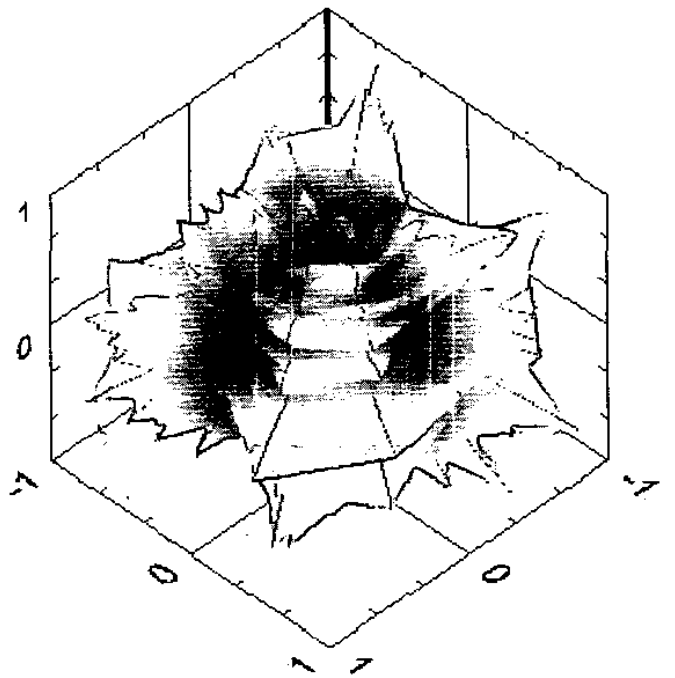

Figure 15. Measured spherical pattern of our artifact at 3 GHz. 


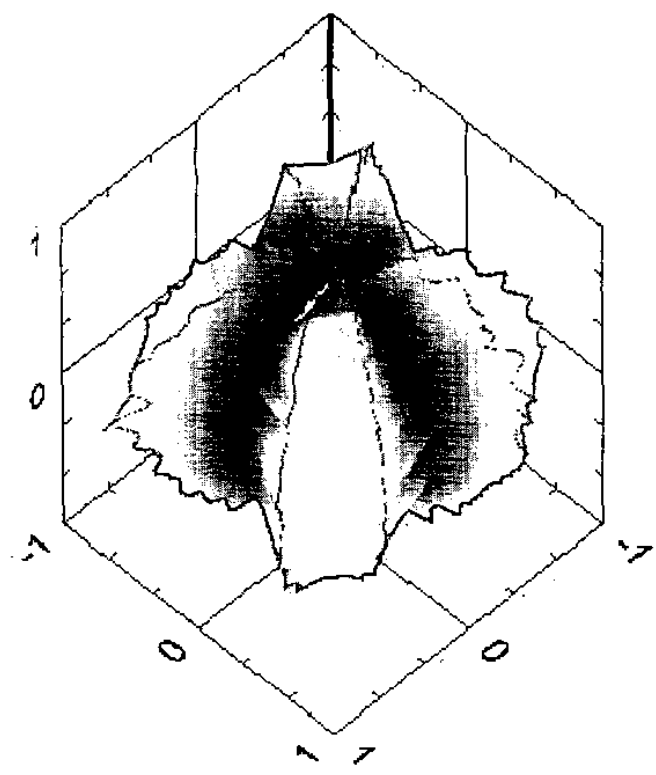

Figure 16. Measured spherical pattern of our artifact, averaged over frequency

tuations are lumped together. Such an approach would ignore the fact that our artifact would be more susceptible when corners rather than surfaces were illuminated. This could be the reason that other researchers $[2-4]$ have found that electrically large devices do not behave like RCs. A purely deterministic approach is doomed due to the extreme sensitivity to configuration. A combined approach, however, appears to give simple models that are able to predict the behavior of complex systems.

\section{CONCLUSIONS}

We have shown that traditional deterministic measurement methods are not practical for electrically large devices, due to large and unpredictable variations and extreme sensitivity to configuration. However, by separating the problem into deterministic and statistical components, we can obtain simpler and more useful models. We find that pattern characteristics can be described by a simple coupling model combined with a reverberation-chamber-type statistical model. The combined model can be used to predict the behavior of a real electrically large system.

\section{ACKNOWLEDGMENTS}

The Systems EM Effects Branch (J52) of the Naval Surface Warfare Center, Dahlgren Division, funded this work. We thank Mike Hatfield of NSWC for supporting this project and also for designing and constructing our artifact. We also thank Katie MacReynolds, Jeff Guerrieri, and Doug Tamura for their help in setting up our artifact on the cylindrical scanning range.

\section{REFERENCES}

[1] L. R. Arnaut and P. D. West, "Evaluation of the NPL untuned stadium reverberation chamber using mechanical and electronic stirring techniques," NPL, CEM 11, 1998.

[2] S. Silfverskiöld, M. Bäckström, and J. Lorén, "Microwave field-to-wire coupling measurements in anechoic and reverberation chambers," IEEE Trans. Electromagn. Compat., vol. 44, p. 233, Feb. 2002.

[3] L. Jansson and M. Bäckström, "Directivity of equipment and its effect on testing in mode-stirred and anechoic chamber," in Proc. IEEE 1999 Int. Symp. EMC, Seattle, WA, Aug. 2-6, 1999, p. 17.

[4] R. St. John and R. Holland, "Field-component statistics of externally illuminated, overmoded cavities," IEEE Trans. Electromagn. Compat., vol. 42, pp. 125134, May 2000.

[5] J. Ladbury, G. Koepke, and D. Camell, "Evaluation of the NASA Langley Research Center mode-stirred chamber facility," NIST, 1508, 1999.

[6] J. Ladbury and K. Goldsmith, "Reverberation chamber verification procedures, or, how to check if your chamber ain't broke and suggestions on how to fix it if it is," in Proc. IEEE 2000 Int. Symp. EMC, Washington, DC, Aug. 21-25, 2000, p. 17.

[7] D. A. Hill, M. T. Ma, A. R. Ondrejka, B. F. Riddle, M. L. Crawford, and R. T. Johnk, "Aperture excitation of electrically large, lossy cavities," IEEE Trans. Electromagn. Compat., vol. 36, pp. 169-178, Aug. 1994.

[8] G. James, Geometrical theory of diffraction for electromagnetic waves. Stevenage: Peter Perigrinus; 1976. 